\title{
Sublingual Nitroglycerin Administration in Coronary Computed Tomography Angiography: a Systematic Review
}

\author{
Richard A. P. Takx ${ }^{1,2}$ • Dominika Suchá ${ }^{2}$. Jakob Park ${ }^{1,3} \cdot$ Tim Leiner $^{2} \cdot$ Udo Hoffmann ${ }^{1}$
}

Received: 4 January 2015 /Revised: 30 March 2015 / Accepted: 9 April 2015 / Published online: 21 May 2015

(C) The Author(s) 2015. This article is published with open access at Springerlink.com

\begin{abstract}
Objective To systematically investigate the literature for the influence of sublingual nitroglycerin administration on coronary diameter, the number of evaluable segments, image quality, heart rate and blood pressure, and diagnostic accuracy of coronary computed tomography (CT) angiography.

Methods A systematic search was performed in PubMed, EMBASE and Web of Science. The studies were evaluated for the effect of sublingual nitroglycerin on coronary artery diameter, evaluable segments, objective and subjective image quality, systemic physiological effects and diagnostic accuracy. Due to the heterogeneous reporting of outcome measures, a narrative synthesis was applied.

Results Of the 217 studies identified, nine met the inclusion criteria: seven reported on the effect of nitroglycerin on coronary artery diameter, six on evaluable segments, four on image quality, five on systemic physiological effects and two on diagnostic accuracy. Sublingual nitroglycerin administration resulted in an improved evaluation of more coronary segments, in particular, in smaller coronary branches, better image quality and improved diagnostic accuracy. Side effects were mild and were alleviated without medical intervention.
\end{abstract}

Richard A. P. Takx

rtakx@umcutrecht.nl

1 Cardiac MR PET CT Program, Department of Radiology, Massachusetts General Hospital, Harvard Medical School, Boston, MA, USA

2 Department of Radiology, University Medical Center Utrecht, Heidelberglaan 100, P.O. Box 85500, 3584

CX Utrecht, The Netherlands

3 Department of Cardiology, University of Heidelberg, Heidelberg, Germany
Conclusion Sublingual nitroglycerin improves the coronary diameter, the number of assessable segments, image quality and diagnostic accuracy of coronary CT angiography without major side effects or systemic physiological changes.

Key Points

- Sublingual nitroglycerin administration results in significant coronary artery dilatation.

- Nitroglycerin increases the number of evaluable coronary branches.

- Image quality is improved the most in smaller coronary branches.

- Nitroglycerin increases the diagnostic accuracy of coronary CT angiography.

- Most side effects are mild and do not require medical intervention.

Keywords Nitroglycerin · Computed tomography · Coronary CT angiography $\cdot$ Coronary vasodilation $\cdot$ Coronary artery disease

\section{Introduction}

Coronary computed tomography angiography (CCTA) has become a useful tool for the exclusion of significant coronary artery disease (CAD) [1-3]. CT allows for image acquisition with high spatial and temporal resolution. Nonetheless, it can be challenging to visualize coronary branches with a small diameter [4]. In clinical practice, nitroglycerin is applied for rapid coronary vasodilation, without causing significant changes in heart rate or blood pressure [5]. Nitroglycerin generates nitric oxide, which initiates smooth muscle relaxation independent of endothelial function [6]. Nitroglycerin also reduces the likelihood of coronary artery vasospasm. Nitroglycerin is widely administered to alleviate angina 
pectoris and has a half-life of about 5 minutes [7, 8]. Sublingual spray is the preferred method of administration since it is the most efficacious and is associated with fewer side effects $[9,10]$. In acute myocardial infarction, sublingual nitroglycerin rapidly relieves pulmonary congestion and decreases myocardial oxygen consumption, although administration might result in a decline in cardiac output [11]. Headache and dizziness are most frequently associated with the use of nitrates [9]. Headaches following the use of sublingual nitroglycerin are fairly common, but are generally short-lasting and self-limiting. Dizziness is typically the result of nitroglycerin-induced hypotension. More serious side effects can occur in patients with diminished cardiac output/severe hypotension, in patients with increased intracranial pressure or patients using phosphodiesterase inhibitors [9, 12]. Feldman et al. [5] demonstrated in invasive coronary angiography that small doses of nitroglycerin result in significant coronary artery and collateral dilatation, without significant changes in heart rate and blood pressure. The administration of nitrates was recommended for conventional coronary angiography by the American College of Cardiology/ American Heart Association (ACC/AHA) in 1999, because it allowed for reliable assessment of coronary stenosis degree $[13,14]$. Subsequently, sublingual nitroglycerin has been routinely used to dilate coronary arteries in CCTA examinations to optimize visualization of the coronary artery lumen and thus improve stenosis assessment $[3,15,16]$.

Multiple studies evaluated the use of sublingual nitroglycerin in CCTA, however the evidence has not been systematically reviewed. This systematic review intended to summarize the current evidence on the effects of sublingual nitroglycerin on coronary diameter, the number of evaluable segments, image quality, heart rate and blood pressure, and diagnostic accuracy of CCTA.

\section{Materials and methods}

\section{Literature search}

To identify all potentially relevant studies, a systematic search was performed using predefined criteria. PubMed, EMBASE and Web of Science were searched to December 2014. The databases were searched using the subject headings "sublingual nitroglycerin" or "nitroglycerin" or "nitrate" or "nitric oxide donors" and "computed tomography" or "CT" or "CCTA" and "coronary" or "cardiac". No search restrictions were applied. A manual reference check of included articles was performed to identify potential studies missed by our search strategy.

\section{Eligibility Criteria}

Article eligibility was determined using predefined criteria. Articles were included based on the following criteria: study domain, patients with suspected or known CAD; index test, CT; evaluation of premedication, nitroglycerin; study results, coronary artery diameter, evaluable segments, objective and subjective (e.g., Likert scale) image quality, systemic physiological effects and diagnostic accuracy. When studies stemmed from overlapping populations, the study with the largest population was included. Animal studies and phantom studies were excluded.

\section{Data Extraction and Analysis}

Data were extracted by two reviewers. CT hardware, study design, number of patients, use of $\beta$-blockers, heart rate, nitroglycerin dose and time between scan were recorded and summarized. Due to the heterogeneous reporting of outcome measures for each study, a formal meta-analysis was not possible. We thus applied a narrative synthesis of the effect of sublingual nitroglycerin on coronary artery diameter, evaluable segments, objective and subjective image quality, systemic physiological effects and diagnostic accuracy.

\section{Results}

\section{Literature search and assessment}

The initial search identified 217 papers after removal of duplicates (Fig. 1). After title/abstract screening, 27 studies remained for full text assessment. Eighteen papers were excluded because they did not meet the predefined selection criteria, yielding a total of nine studies.

\section{Study characteristics}

The study characteristics of the included studies are listed in Table 1. Of these, seven reported on the effect of nitroglycerin on coronary artery diameter, six on evaluable segments, four on image quality, five on systemic physiological effects and two on diagnostic accuracy.

\section{Coronary artery diameter}

Seven studies evaluated the effect of sublingual nitroglycerin on coronary diameter on CCTA. Two studies used a randomized design [20,23], one used intra-individual comparison $[17,25]$, one used a matched design [22] and two used a retrospective review of their database [18, 24]. All studies demonstrated an overall increase in diameter with the use of 
Fig. 1 Flow chart. Search performed on December 23, 2014

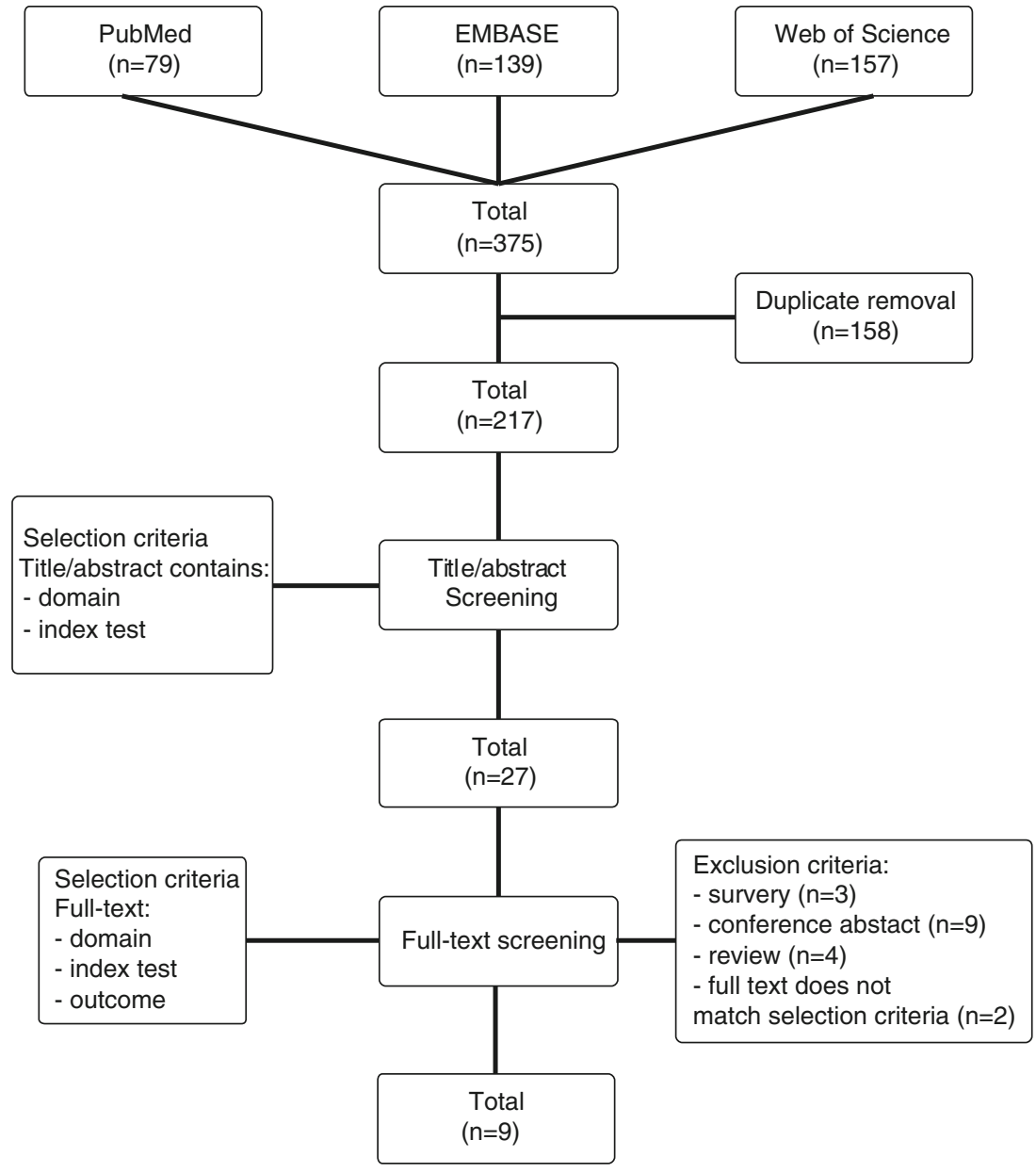

nitroglycerin, ranging from 8 to $30 \%$ (Table 2). Klass et al. [22] showed that the relative vessel diameter increase is larger in distal segments compared to proximal segments. Okada et al. [25] and Sato et al. [23] confirmed this observation for peripheral segments. Kang et al. [18] found a relative increase in diameter in two patients who underwent CCTA with and without administration of nitroglycerin.

\section{Evaluable segments}

Six CCTA studies evaluated the effect of sublingual nitroglycerin on the number of evaluable segments/branches [18-20, 23-25]. All studies showed an improvement in the number of evaluable segments in the nitroglycerin group. Okada et al. [25] observed a non-significant increase in their intraindividual comparison in 52 patients. The effect was particularly profound when only comparing the number of side branches, as was performed by Decramer et al. [20]. Lee et al. [24] found a significant improvement in the visibility of second order branches of the left anterior descending (LAD) artery and the right coronary artery (RCA). Chun et al. [19] showed that the application of nitroglycerin resulted in significantly more evaluable distal segments.

\section{Image quality}

Two studies found that the administration of sublingual nitroglycerin resulted in significantly better subjective image quality, especially for the posterior descending artery [22, 24]. Decramer et al. [20] did not observe an effect of nitroglycerin on objective measures such as signal-to-noise and contrast-tonoise ratio, nor did they observe a difference in the number of scans with substantial artefacts with the use of sublingual nitroglycerin. Okada et al. [25] did not find a significant difference in subjective image quality. Two studies observed significantly higher intraluminal attenuation in the nitroglycerin group for the more peripheral vessels [22, 25].

\section{Systemic physiological effects}

Three studies observed no significant changes in blood pressure and/or heart rate [19-21]. Two more extensive studies did observe a significant change in heart rate, though heart rate variability tended to be lower in the group that received nitroglycerin $[23,25]$. Okado et al. also observed a notable decrease in systolic $(10.6 \%)$ and diastolic $(9.1 \%)$ blood pressure. Sato et al. [23] found significant changes in 

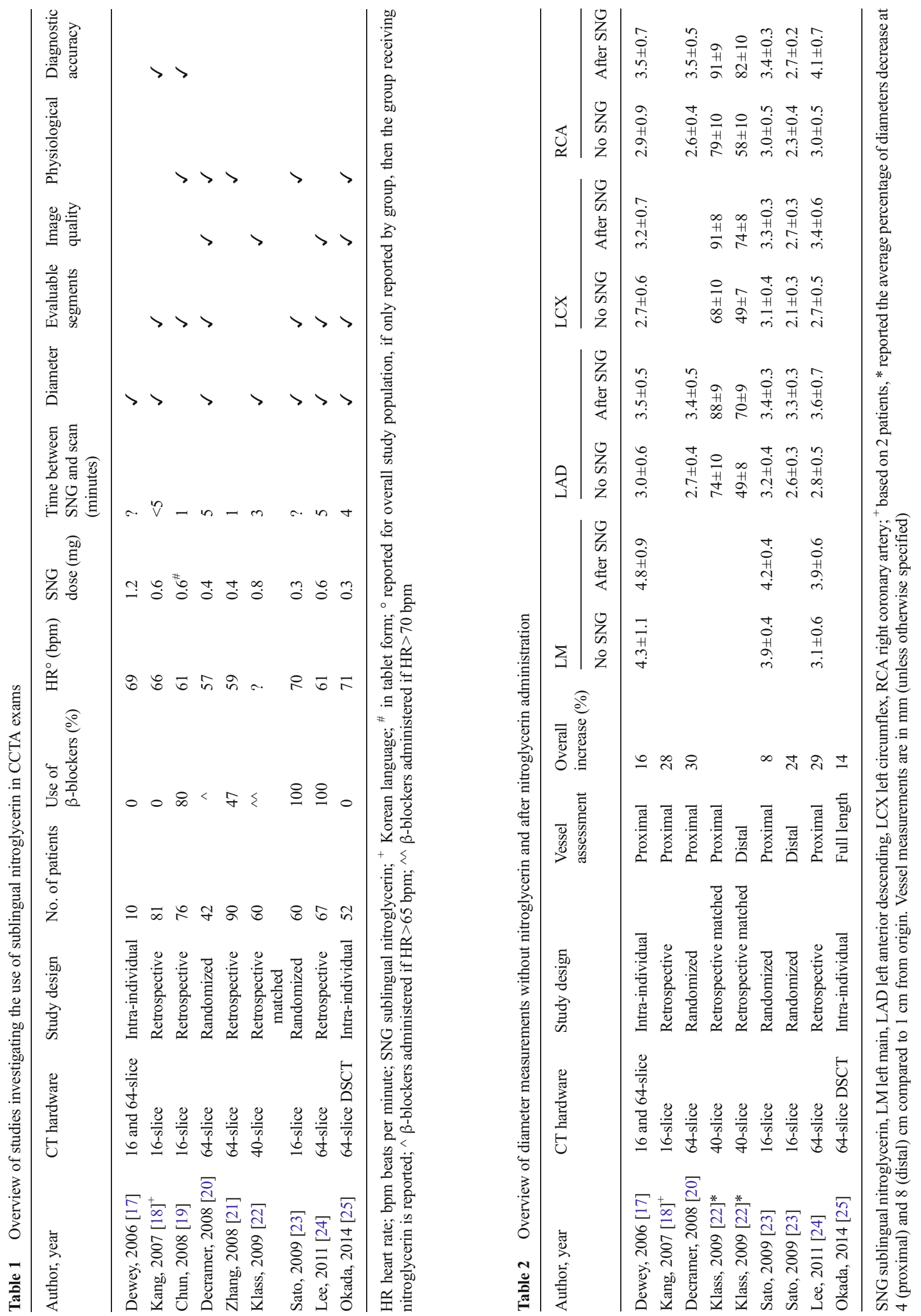
hemodynamics after 4 minutes. Only a few studies reported on side effects; all were mild (e.g., headache or dizziness) and were alleviated without medical intervention [19, 20, 23, 24].

\section{Diagnostic accuracy}

The effect of sublingual nitroglycerin on diagnostic performance was evaluated in two studies using 16-detector row CT $[18,19]$. Sensitivity and specificity were evaluated for a $\geq 50 \%$ stenosis degree. Chun et al. [19] reported a sensitivity of $97.0 \%$ for patient-based evaluation with a specificity of $84.6 \%$ for the nitroglycerin group compared to $90.0 \%$ and $70.0 \%$, respectively, for the control group. Diagnostic accuracies for proximal, mid, and distal segments were all higher in the patients who received sublingual nitroglycerin. Overall sensitivity for segment-based evaluation was $83.0 \%$ with a specificity of $97.8 \%$ for the nitroglycerin group compared to $63.6 \%$ and $94.8 \%$, respectively, for the control group. Kang et al. [18] determined the diagnostic accuracy in a subset of 42 patients who underwent invasive coronary angiography. On a segment basis, they found a comparable diagnostic accuracy of $79.5 \%$ for the nitroglycerin group and $84.1 \%$ for the control group. The diagnostic accuracy for finding any atherosclerosis was higher in the nitroglycerin group ( $85.8 \%$ vs. $80.1 \%)$.

\section{Discussion}

This systematic review provides an overview of the current evidence on the effect of sublingual nitroglycerin. We found that one to two pumps of sublingual nitroglycerin $(0.4-0.8 \mathrm{mg})$ increased coronary diameter, the number of assessable coronary artery segments, image quality and diagnostic accuracy of CCTA. The administration of nitroglycerin seems to be especially beneficial in smaller coronary branches. We observed among the included studies an increase in the number of evaluable segments as well as an increase in subjective and objective image quality in smaller coronary branches. The spatial resolution of CT may not be sufficiently high to visualize the smallest coronary branches; nevertheless nitroglycerin administration is able to increase the number of evaluable segments.

Sato et al. [23] recorded heart rate and blood pressure every minute during image acquisition and observed significant changes in systemic hemodynamics after 4 minutes. However, only one scan acquisition was acquired and, thus, evaluation of maximal coronary dilatation was not feasible. Pepe et al. [26] observed a maximal vasodilator response between 4 and 5 minutes after nitroglycerin administration using magnetic resonance imaging (MRI) for visualization of the coronary artery lumen. Also, they noted among their subjects substantial heterogeneity in the time point of maximal coronary dilatation. On the basis of these studies, the optimal starting time for image acquisition is probably between 3 and 4 minutes after sublingual administration of nitroglycerin.

A point of concern is that the administration of nitroglycerin could result in overestimation of the stenosis degree [27]. Conti et al. [28] showed in 47 patients (119 coronary artery stenoses) undergoing conventional angiography that the use of sublingual nitroglycerin results in a significant increase in percentual stenosis $(8 \%$ mean) and that the more narrowed stenoses were less likely to dilate. This finding could be explained by a decrease in vascular reactivity to nitroglycerin in diseased vessels, especially in atherosclerotic segments with circumferential disease [29]. Another point of concern with the administration of nitroglycerin is that it can result in a decrease in blood pressure or reflex tachycardia $[9,30]$. Reflex tachycardia, although rare, could result in more motion artefacts on CCTA [30]. Only Decramer et al. [20] evaluated the effect of nitroglycerin on the presence of substantial artefacts and did not observe significant differences.

The applicability of nitroglycerin in certain subgroups is of interest. Females have smaller coronary arteries compared to males after controlling for differences in body size [31]. Moreover, oestrogen affects vascular tone through production of nitric oxide by the endothelium [32,33]. However, none of the included studies investigated the effect of nitroglycerin in women compared to men. Another potentially challenging population are hypertensive patients, although in a small study using invasive angiography, no significant change in the lumen area was observed between normotensive and hypertensive patients after administration of sublingual nitroglycerin [34].

An interesting new field is the effect of nitroglycerin on the diagnostic accuracy of fractional flow reserve $\mathrm{CT}\left(\mathrm{FFR}_{\mathrm{CT}}\right)$. Advances in computational fluid dynamics allow for calculation of hemodynamic significance of coronary stenoses in CCTA examinations, which is based on an anatomic model of the coronaries generated from CCTA data [35]. In the DeFACTO study [36], the effect of sublingual nitroglycerin on diagnostic accuracy of $F F R_{C T}$ was evaluated. Administration of nitroglycerin was linked to improved specificity of $\mathrm{FFR}_{\mathrm{CT}}(55.2 \%$ vs. $65.0 \%)$. Improvement in the diagnostic accuracy of $\mathrm{FFR}_{\mathrm{CT}}$ was most profound when nitroglycerin was given 30 minutes or less before the CCTA examination (67.5\% vs. $78.6 \%$ ), which underscores the importance of early image acquisition after administration of sublingual nitroglycerin.

A limitation of this systematic review was that many studies used 16-slice CT, which is considered suboptimal for coronary imaging due to limited temporal resolution compared to current CT scanners. Second, only two studies evaluated the effect of nitroglycerin using an intra-individual comparison. Third, the time between sublingual nitroglycerin administration and scan acquisition was not listed in all of the included 
studies. Finally, we were not able to meta-analyze our findings due to the heterogeneous reporting of outcome measures.

In conclusion, despite its widespread use in clinical practice, only a few comprehensive studies evaluating the effect of nitroglycerin in coronary CCTA have been published. Our systematic review of the available evidence on the use of sublingual nitroglycerin in CCTA suggests that nitroglycerin improves coronary diameter, the number of assessable segments, image quality, and diagnostic accuracy, although only a few studies met the inclusion criteria. Only mild systemic physiological changes which may impact image quality can be expected if scan acquisition is performed within 4 minutes after nitroglycerin administration. However, the vasodilatory effects of nitroglycerin are reduced in atherosclerotic coronary segments. More studies are needed to assess the effect of nitroglycerin on stenosis assessment and $\mathrm{FFR}_{\mathrm{CT}}$ measurements, especially when CCTA is performed on current generation scanners.

Acknowledgments The scientific guarantor of this publication is Tim Leiner. The authors of this manuscript declare no relationships with any companies whose products or services may be related to the subject matter of the article. Jakob Park received funding from the German Cardiac Society and German National Academic Foundation. All other authors have nothing to declare. No complex statistical methods were necessary for this paper. Institutional Review Board approval and written informed consent were not necessary because the study was a systematic review. Some study subjects or cohorts have been previously reported. This study is a systematic review; as such, this is a summary of previously published material.

Open Access This article is distributed under the terms of the Creative Commons Attribution-NonCommercial 4.0 International License (http:// creativecommons.org/licenses/by-nc/4.0/), which permits any noncommercial use, distribution, and reproduction in any medium, provided you give appropriate credit to the original author(s) and the source, provide a link to the Creative Commons license, and indicate if changes were made.

\section{References}

1. Hulten EA, Carbonaro S, Petrillo SP, Mitchell JD, Villines TC (2011) Prognostic value of cardiac computed tomography angiography: a systematic review and meta-analysis. J Am Coll Cardiol 57:1237-1247

2. Dharampal AS, Papadopoulou SL, Rossi A et al (2013) Diagnostic performance of computed tomography coronary angiography to detect and exclude left main and/or three-vessel coronary artery disease. Eur Radiol 23:2934-2943

3. Maurer MH, Zimmermann E, Schlattmann P, Germershausen C, Hamm B, Dewey M (2012) Indications, imaging technique, and reading of cardiac computed tomography: survey of clinical practice. Eur Radiol 22:59-72

4. Martuscelli E, Romagnoli A, D'Eliseo A et al (2004) Accuracy of thin-slice computed tomography in the detection of coronary stenoses. Eur Heart J 25:1043-1048

5. Feldman RL, Pepine CJ, Curry RC Jr, Conti CR (1979) Coronary arterial responses to graded doses of nitroglycerin. Am J Cardiol 43: 91-97
6. Ignarro LJ, Napoli C, Loscalzo J (2002) Nitric oxide donors and cardiovascular agents modulating the bioactivity of nitric oxide: an overview. Circ Res 90:21-28

7. Parker JD, Parker JO (1998) Nitrate therapy for stable angina pectoris. N Engl J Med 338:520-531

8. Armstrong PW, Armstrong JA, Marks GS (1979) Blood levels after sublingual nitroglycerin. Circulation 59:585-588

9. Thadani U, Rodgers T (2006) Side effects of using nitrates to treat angina. Expert Opin Drug Saf 5:667-674

10. Pfister M, Seiler C, Fleisch M, Gobel H, Luscher T, Meier B (1998) Nitrate induced coronary vasodilatation: differential effects of sublingual application by capsule or spray. Heart 80:365-369

11. Williams DO, Amsterdam EA, Mason DT (1975) Hemodynamic effects of nitroglycerin in acute myocardial infarction. Circulation $51: 421-427$

12. Ahmad S (1991) Nitroglycerin and intracranial hypertension. Am Heart J 121:1850-1851

13. Jost S, Rafflenbeul W, Reil GH et al (1990) Reproducible uniform coronary vasomotor tone with nitrocompounds: prerequisite of quantitative coronary angiographic trials. Cathet Cardiovasc Diagn 20:168-173

14. Scanlon PJ, Faxon DP, Audet AM et al (1999) ACC/AHA guidelines for coronary angiography. A report of the American College of Cardiology/American Heart Association Task Force on practice guidelines (Committee on Coronary Angiography). Developed in collaboration with the Society for Cardiac Angiography and Interventions. J Am Coll Cardiol 33:1756-1824

15. Maurer MH, Hamm B, Dewey M (2009) Survey regarding the clinical practice of cardiac CT in Germany: indications, scanning technique and reporting. Röfo 181:1135-1143

16. Johnson PT, Eng J, Pannu HK, Fishman EK (2008) 64-MDCT angiography of the coronary arteries: nationwide survey of patient preparation practice. AJR Am J Roentgenol 190:743-747

17. Dewey M, Hoffmann H, Hamm B (2006) Multislice CT coronary angiography: effect of sublingual nitroglycerine on the diameter of coronary arteries. Röfo 178:600-604

18. Kang DK, Noh HW, Park KJ, Choi SY (2007) Assessment of the image quality and diagnostic accuracy of coronary CT angiography: effect of sublingual administration of nitroglycerin. J Korean Radiol Soc 56:127-135

19. Chun EJ, Lee W, Choi YH et al (2008) Effects of nitroglycerin on the diagnostic accuracy of electrocardiogram-gated coronary computed tomography angiography. J Comput Assist Tomogr 32:86-92

20. Decramer I, Vanhoenacker PK, Sarno G et al (2008) Effects of sublingual nitroglycerin on coronary lumen diameter and number of visualized septal branches on 64-MDCT angiography. AJR Am J Roentgenol 190:219-225

21. Zhang J, Fletcher JG, Harmsen WS et al (2008) Analysis of heart rate and heart rate variation during cardiac $\mathrm{CT}$ examinations. Acad Radiol 15:40-48

22. Klass O, Mutlu S, Hohl K et al (2009) Multidetector computed tomography coronary angiography: sublingual nitroglycerine improves image quality significantly because of peripheral coronary vasodilatation. J Comput Assist Tomogr 33:199-203

23. Sato K, Isobe S, Sugiura K et al (2009) Optimal starting time of acquisition and feasibility of complementary administration of nitroglycerin with intravenous beta-blocker in multislice computed tomography. J Comput Assist Tomogr 33:193-198

24. Lee CM, Wang HJ, Kung CH et al (2011) Evaluation of nitroglycerin premedication on the arterial luminal diameter and branches of coronary arteries on 64-multidetector computed tomography angiography. J Exp Clin Med 3:85-88

25. Okada M, Nakashima Y, Nomura T et al (2014) Coronary vasodilation by the use of sublingual nitroglycerin using 64-slice dualsource coronary computed tomography angiography. J Cardiol. doi:10.1016/j.jjcc.2014.05.012 
26. Pepe A, Lombardi M, Takacs I, Positano V, Panzarella G, Picano E (2004) Nitrate-induced coronary vasodilation by stress-magnetic resonance imaging: a novel noninvasive test of coronary vasomotion. J Magn Reson Imaging 20:390-394

27. Hoffmann U, Ferencik M, Cury RC, Pena AJ (2006) Coronary CT angiography. J Nucl Med 47:797-806

28. Conti CR, Feldman RL, Pepine CJ, Hill JA, Conti JB (1983) Effect of glyceryl trinitrate on coronary and systemic hemodynamics in man. Am J Med 74:28-32

29. Yamagishi M, Nissen SE, Booth DC et al (1995) Coronary reactivity to nitroglycerin: intravascular ultrasound evidence for the importance of plaque distribution. J Am Coll Cardiol 25:224-230

30. Khan M, Cummings KW, Gutierrez FR, Bhalla S, Woodard PK, Saeed IM (2011) Contraindications and side effects of commonly used medications in coronary $\mathrm{CT}$ angiography. Int $\mathrm{J}$ Cardiovasc Imaging 27:441-449

31. Sheifer SE, Canos MR, Weinfurt KP et al (2000) Sex differences in coronary artery size assessed by intravascular ultrasound. Am Heart J 139:649-653
32. Knot HJ, Lounsbury KM, Brayden JE, Nelson MT (1999) Gender differences in coronary artery diameter reflect changes in both endothelial Ca2+ and ecNOS activity. Am J Physiol 276:H961-H969

33. Wellman GC, Bonev AD, Nelson MT, Brayden JE (1996) Gender differences in coronary artery diameter involve estrogen, nitric oxide, and $\mathrm{Ca}(2+)$-dependent $\mathrm{K}+$ channels. Circ Res 79:1024-1030

34. Frielingsdorf J, Kaufmann P, Seiler C, Vassalli G, Suter T, Hess OM (1996) Abnormal coronary vasomotion in hypertension: role of coronary artery disease. J Am Coll Cardiol 28:935-941

35. Grunau GL, Min JK, Leipsic J (2013) Modeling of fractional flow reserve based on coronary $\mathrm{CT}$ angiography. Curr Cardiol Rep 15: 336

36. Leipsic J, Yang T-H, Thompson A et al (2014) CT Angiography (CTA) and diagnostic performance of noninvasive fractional flow reserve: results from the determination of fractional flow reserve by anatomic CTA (DeFACTO) study. Am J Roentgenol 202:989-994 